\title{
Current Status in the Surgery and Conservative Management of Upper Urinary System Tumors
}

\author{
- Serdar Geyik MD \\ Private Avrupa Hospital, Clinic of Urology, Adana, Turkey
}

\begin{abstract}
Upper urinary tract urothelial cancers constitute 5\% of all urothelial cancers and standard therapy is bladder cuff excision with nephroureterectomy This treatment is performed as open or technologically assisted (laparoscopic and robotic) surgery. Conservative treatment approaches include endoscopic treatments, segmental resection, and upper urinary tract immunotherapy. Upper urinary tract immunotherapy is considered effective in patients with carcinoma in situ. In addition, the development of targeted molecules and gene therapies is also anticipated. In selected patient groups, endoscopic (antegrade percutaneous and retrograde ureteroscopic) procedures are being implemented as an alternative to nephroureterectomy in order to prevent unnecessary organ loss and to preserve the nephrons. Oncological survival and tumor control with the endoscopic approach are similar to the outcomes of radical surgery in cases with low-stage and grade tumors. Advantages of the endoscopic approach include minimal morbidity and preservation of kidney function, while disadvantages include life-long follow-up and the need for a large number of endoscopies. In cases with a large tumor burden, the retrograde ureteroscopic approach is generally preferred instead of percutaneous treatment.
\end{abstract}

Keywords: Upper urinary system urothelial cancers, conservative treatment, alternative surgical approaches, oncologic survival, tumor control

\section{Introduction}

Urothelial carcinoma is the $4^{\text {th }}$ most common cancer after breast (or prostate), lung, and colorectal cancers (1). Approximately $90-95 \%$ of urothelial carcinomas originate in the bladder and $5-10 \%$ in the upper urinary tract (UUT) (2). Seventeen percent of patients with UUT tumors (UUTTs) have concomitant bladder tumors (3). According to the European Association of Urology (EAU) guidelines, standard methods for the diagnosis of UUTTs include:

- Urine cytology,

- Cystoscopy,

- Computed tomography (CT),

- Urography,

- Ureteroscopy and biopsy (3).

Recent reports have cited CT urography as the best noninvasive method for evaluating the UUT for urothelial malignancies. There has been debate regarding the timing and method of contrast media administration, as well as imaging criteria used to identify suspected malignancy (4).

Other than these standard diagnostic methods, there are additional diagnostic methods to increase diagnostic potential in cases of carcinoma in situ (CIS) and small flat lesions, although their effectiveness has not been established. Narrow band imaging (NBI) and photodynamic diagnosis (PDD) are used. $\mathrm{NBI}$ is based on the principle of increasing contrast between the mucosa and tumor tissues using light at 2 different wavelengths (blue and green) (5). The method was reported in 1 study to increase the diagnosis rate by $23 \%$ (6). Similarly, PDD works on the principle that when a fluorochrome (5-aminolevulinic acid) accumulates in tissue, the pink pathological tissues appear on a blue background when viewed under a light source with a specific wavelength. Two studies on this subject reported that more tumors were detected $(7,8)$. Although further validation of their effectiveness is necessary, these modalities are promising for the future.

The prevalence of invasive tumors at initial diagnosis is $15-25 \%$ for the bladder and $60 \%$ in the UUT (9). In recurrences of UUTTs, $22-47 \%$ of tumors are detected in the bladder, and $26 \%$ in the UUT (10). The prevalence of UUTT is 3 times greater in males and peaks in the 70-90 year age group (11). Similarly, tumor grade can be determined with $90 \%$ accuracy with ureterorenoscopic biopsy, independent of tumor size (12).

Address for Correspondence: Serdar Geyik MD, Private Avrupa Hospital, Clinic of Urology, Adana, Turkey

E-mail: srdrgeyik@gmail.com ORCID-ID: orcid.org/0000-0002-8712-7682

Received: 16.07.2018 Accepted: 31.07.2018 
Examination of resection (nephroureterectomy) material from patients with pelvic tumors in the UUT showed that CIS and dysplasia in different locations were present in $60-95 \%$ of cases, and recurrences were ultimately the result of a tendency toward multifocal localization (13). Due to this localization pattern, the standard treatment for UUTT is radical nephroureterectomy (RNU). The treatment algorithm is shown in Table 1.

The most important parameter affecting treatment outcomes of UUTT is tumor stage and grade. As with bladder cancers, spread occurs via hematogenous and lymphatic route or direct invasion. Muscle invasion has a direct impact on 5-year survival ( $\leq 50 \%$ among patients with T2-T3 tumors versus $<10 \%$ for those with T4 tumors) (14).

The classification of patients into low- and high-risk groups is crucial when deciding between conservative and radical surgery in cases of UUTT (15). The determination of low- and highrisk factors is presented in Table 2 . The presence of any of the

Table 1. Treatment algorithm for upper urinary tract tumors in the 2015 European Association of Urology Guidelines

UPPER URINARY TRACT UROTHELIAL CARCINOMA

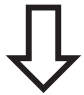

CT UROGRAPHY, CYTOLOGY, CYSTOSCOPY, RETROGRADE PYELOGRAPHY
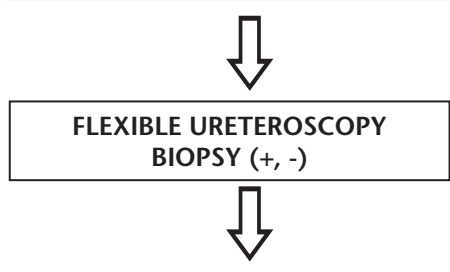

\begin{tabular}{|l|}
\hline - SINGLE TUMOR FOCUS \\
- SMALL TUMOR $(<1 \mathrm{CM})$ \\
- LOW GRADE \\
- NONINVASIVE CT APPEARANCE \\
- PATIENT COMPLIANCE WITH \\
CLOSE FOLLOW-UP \\
\hline
\end{tabular}

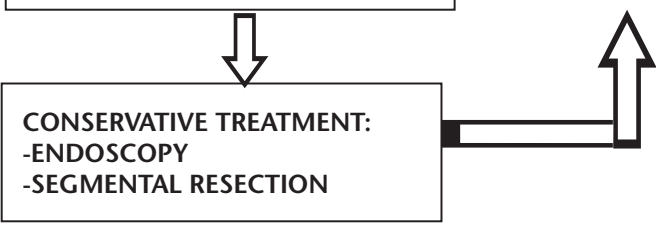

CT: Computed tomography high-risk factors is sufficient for the patient to be classified in the high-risk group. The gold standard treatment for high-risk tumors is RNU with cuff excision. Conservative treatment of UUTTs is an option for low-risk patients and special indications (such as solitary kidney and renal failure) $(3,15)$.

According to version 4.2018 of the National Comprehensive Cancer Network guidelines for bladder cancer, nephron-sparing endoscopic (ureteroscopic and percutaneous approaches) interventions can be recommended alone or in combination with postoperative intrapelvic adjuvant therapies or bacillus Calmette-Guérin (BCG) for low-risk patients. Ureteroscopic methods or resection and anastomosis of the upper/middle/ lower ureter can be performed when selecting endoscopic interventions for the renal pelvis and upper ureter (16). However, the risk of tumor spread along the access path should be kept in mind when using percutaneous approaches. In terms of postoperative follow-up, EAU guidelines recommend endoscopy, cytology, and CT urography at 3 and 6 months, every 6 months for the following 2 years, and once a year thereafter for a total of at least 5 years (17).

Open, Laparoscopic, and RoboticRadical Nephroureterectomy and Distal Ureterectomy Techniques

The nephrectomy portion of radical surgery for UUTTs has been standardized in terms of surgical principles, regardless of the technique used. In contrast, many different techniques for distal ureterectomy and cuff excision surgery have been developed to date, some of which have been abandoned due to recurrence and difficulties. Some surgical techniques are practiced in combination based on the patient's condition and the surgeon's experience and preference. The following sections provide an outline of the techniques utilized in the surgical treatment of UUTTs.

\section{Open Radical Nephroureterectomy}

Single or double incisions may be made based on surgeon experience and the patient's physical build, kidney size, and affected side. For a single incision, a thoracoabdominal incision extending below the umbilicus should be preferred. If a double incision is planned, then a flank incision plus a Gibson or Pfannenstiel incision can be made. It is important to rule out the presence of a second artery and to excise the renal fascia and surrounding adipose tissue before ligating the renal vein. Adrenalectomy is recommended in patients with locally advanced disease and intraoperative signs of adrenal involvement. Cuff resection may be performed transvesically and/or extravesically, and a combined approach with endoscopy provides a comfortable surgery. It is important to remove the ureter as a whole and to close the bladder in 2 layers (18).

Table 2. Classification of high- and low-risk factors in upper urinary tract tumors in the 2015 European Association of Urology Guidelines

\begin{tabular}{|l|l|l|l|l|l|l|l|}
\hline Risk factor & Cytology & Number of foci & Tumor size & Pathology & Hydronephrosis & Invasion on CT urography & $\begin{array}{l}\text { Previous radical } \\
\text { cystectomy }\end{array}$ \\
\hline Low & Low & Single & $<1 \mathrm{~cm}$ & Low & $(-)$ & $(-)$ & $(-)$ \\
\hline High & High & Multiple & $>1 \mathrm{~cm}$ & High & $(+)$ & $(+)$ & $(+)$ \\
\hline \multicolumn{7}{|l|}{ CT: Computed tomography } \\
\hline \multicolumn{7}{|l|}{} \\
\hline
\end{tabular}




\section{Laparoscopic Radical Nephroureterectomy}

First described by Clayman et al. (19) in 1991, the laparoscopic method is preferable for low stage/grade upper ureter or renal pelvis tumors with low risk of invasive disease. Typically, laparoscopic surgery is advantageous in terms of morbidity. Oncologic efficacy and relapse are equivalent to open surgery $(19,20)$. Details to be considered during laparoscopy include avoiding direct contact between instruments and the tumor, avoiding violation of the urinary tract, using organ bags and ensuring en bloc removal of the kidney and ureter cuff, and avoiding laparoscopy in cases of invasive and large tumors ( $\mathrm{T} 3 / 4$ and/or $\mathrm{N}+, \mathrm{M}+)$. The procedure may be retroperitoneal or transperitoneal. Laparoscopy combined with endoscopy facilitates removal of the distal ureter.

Distal ureterectomy and cuff resection are the least standardized part of laparoscopic RNU surgery. The choice is based on surgeon experience, tumor site and potential for spread, and the technical means available (21).

\section{Robotic Radical Nephroureterectomy}

Enables fine manipulations that are not possible with the laparoscopic technique, as well as easier suturing and reconstruction. It is particularly advantageous for distal ureter and cuff removal and bladder suturing. As with the other methods, a Foley catheter is recommended for 1 week. Similarly, adrenalectomy is also recommended in this method in cases with adrenal involvement (22).

Approach to bladder cuff resection: Due to the $30-75 \%$ recurrence rate in ureteral stumps, RNU with cuff excision is the standard surgical approach. Open and technologically assisted (laparoscopic or robotic) surgeries are possible. Open surgery is associated with greater morbidity, and its nonsuperiority over the laparoscopic or robotic approach in terms of oncologic recurrence has been emphasized (23). Only $1 \%$ of UUTTs originate in the ureter. The need for RNU is absolute in the high-risk group, but there is still uncertainty regarding the cuff excision technique and whether it is necessary for all patients (1).

Renal pelvis tumors have a better prognosis than ureteral tumors due to the barrier effect of the renal parenchyma (24). In a multicenter renal pelvis tumor series of 4210 patients, Lughezzani et al. (25) evaluated the outcomes of RNU with cuff excision in 2492 patients and RNU alone in 1718 patients. Among patients with locally advanced disease, they determined that the cancer-related mortality rates of pT3-4/ $\mathrm{N} 0$ and $\mathrm{pT}$ (any)/N1-3 patients were 1.25 times and 1.45 times higher, respectively, when cuff excision was not performed. These findings showed that cuff resection is absolutely necessary for these patients, but not performing cuff excision on patients with localized $\mathrm{pT} 1-2 / \mathrm{N} 0$ renal pelvis tumors does not affect survival; therefore, they concluded that RNU alone can be performed in T1-2 patients (25).

"Pluck" technique (transurethral resection of ureteral orifice): Prior to nephrectomy, the orifice is dissected and freed to the perivesical area via cystoscopy using a holmium laser or resectoscope. This is followed by nephrectomy and the antegrade removal of the ureter without suturing the bladder. A Foley catheter is left place for 1 week to allow the bladder incision to heal. The method is contraindicated in patients with distal ureter tumor or CIS (21).

Stripping (intussusception): No longer recommended in EAU guidelines (3). During nephrectomy, a basket or balloon is placed cystoscopically, after which the ureter is ligated. The remaining distal portion is secured in the balloon or basket and traction is applied to the catheter to draw it into the bladder while stripping the ureter from the periureteral tissue, followed by resection of the orifice. Various technical setbacks (such as ureteral rupture) may arise during the procedure (21).

Cleveland technique (transvesical method): Access to the bladder is achieved through $3 \mathrm{~mm}$ suprapubic ports, the ureteral orifice is sealed with an endoloop, and the orifice is completely circumscribed to the perivesical area. The distal aspect is removed by proximal traction-assisted dissection during nephrectomy. Seeing the complete orifice sealed with the endoloop is an indicator of safe surgery (26).

Total laparoscopic technique: The orifice and its surroundings are first cauterized using a cystoscope. After nephrectomy, antegrade traction is applied to the ureter and it is mobilized towards the distal aspect. The orifice is everted out of the bladder and secured with an endovascular staple. Seeing the cauterized area is an indicator of surgical integrity (21).

Various researchers have described modified patient positions and different robotic arm paths that are advantageous in robotic RNU. Operative times of 150-190 minutes and blood loss of approximately $130 \mathrm{~mL}$ have been reported in different series $(27,28)$. Among more recent publications, Tamhankar et al. (29) reported the average operative time and amount of blood loss in 11 robotic RNU and pelvic lymphadenectomy procedures as 170 minutes (156-270 minutes) and $150 \mathrm{cc}$ (25$500 \mathrm{cc})$, respectively. The mean length of hospital stay was 3 days (2-8 days). With the exception of 1 patient who developed metastatic systemic recurrence during a 9-month follow-up period, all patients were reported to be disease-free in the final follow-up. As a result, they emphasized the operative morbidity advantage of the robotic approach and its reliability in terms of short-term oncological outcomes (29).

A comparison of different distal ureterectomies (pluck, full laparoscopic, robotic) conducted by Stravodimos et al. (30) showed that none of the techniques was superior. However, they recommended open ureterectomy and cuff excision in patients with excessive tumor load in the distal ureter. They authors stated that due to the high risk of intravesical recurrence, the pluck technique is appropriate for low-stage/grade tumors of the proximal ureter and renal pelvis. It was also emphasized that compared to open ureterectomy, the laparoscopic and robotic methods offer easy bladder suturing after cuff resection and are superior in terms of negative surgical margins and high patient tolerance (30). In another study, Hanna et al. (31) conducted a multicenter comparison of surgical outcomes of 7401 open and 754 laparoscopic operations. Although the low rates of blood transfusion and intraoperative complications in the laparoscopic group were noted, the authors emphasized that the groups showed no difference in terms of postoperative complications and that laparoscopy was a superior alternative to open surgery in terms of morbidity (31).

$\mathrm{Li}$ et al. (32) compared cuff resection methods (intravesical, extravesical, transurethral) in 301 patients who underwent RN 
and reported no superiority in terms of recurrence or cancerspecific survival. As recommendations, they emphasized orifice coagulation in the transurethral approach and early ureter clamping during nephrectomy to prevent potential spread (32). In 2 large multicenter studies, recurrence and cancer-specific mortality rates following RNU were compared according to pathology ( $T$ classification, lymph node metastasis, lymphovascular invasion, sessile tumor and CIS presence) and tumor location. In their study encompassing 2244 patients in 23 centers, Cha et al. (33) reported that tumor pathology was associated with recurrence and cancer-specific mortality over 45 months of follow-up. Raman et al. (34) reached the same conclusion in their study including 1249 patients, and they also reported that tumor location in the ureter or pelvis did not affect recurrence or survival. Zou et al. (35) compared open $(n=101)$ and laparoscopic $(n=21)$ nephroureterectomies and reported that surgical technique did not affect intravesical recurrence or mortality, while history of preoperative tumor in the bladder and presence of hydronephrosis were associated with intravesical recurrence, and tumor pathology (stage, grade, and lymphovascular invasion) was a significant predictor of cancer-specific mortality. Two studies with follow-up periods of 5 years (36) and 13.6 years (37) are noteworthy in terms of the long-term follow-up and survival comparisons between laparoscopic and open NU. Neither study was able to demonstrate a significant difference in survival between the surgical techniques.

In conclusion, when techniques pertaining to distal ureter and cuff resection during NU are compared, open ureterectomy and cuff resection are considered the gold standard. Using a laparoscopic stapler may result in positive surgical margin in the bladder; the transvesical laparoscopic approach, while oncologically reliable, has technical difficulties; and the transurethral approach and intussusception carry the risk of tumor seeding. The long-term follow-up results for robotassisted distal ureterectomy are still insufficient.

In terms of lymph node dissection, the rate of positivity in $\mathrm{Ta}$ and $\mathrm{T} 1$ disease is reported as $2.2 \%$ and dissection is not recommended. In contrast, lymph node positivity is reported as about $16 \%$ in T2 and T3 disease, and dissection is recommended in these cases $(3,38)$.

Lee et al. (39) evaluated the relationship between recurrence in the bladder and preoperative ureteroscopic biopsy in patients who underwent RNU and cuff resection secondary to UUTT. Of 502 patients operated between 1990 and 2013, they reported no significant difference in bladder tumor recurrence between those who underwent preoperative ureteroscopic biopsy (206 patients) and those who did not (296 patients). It was emphasized that a history of bladder tumor, the surgical technique used, and primary tumor pathology are influential in postoperative bladder recurrences (39). In the EAU guidelines, a single dose of mitomycin- $C$ instilled before bladder catheter removal was reported to be effective in preventing early bladder recurrence (3).

\section{Conservative or Local Treatment}

Conservative or local treatments are preferred in low-risk patients, those with a functional contralateral kidney, and when other approaches are not an option (solitary kidney or renal failure) (3). When following a conservative treatment approach, the ipsilateral UUT should be monitored closely.

Segmental resections: For proximal and mid-ureter tumors, open segmental ureterectomy is recommended in the following cases:

- Grade 1-2 T2 disease in which preserving renal function is necessary,

- Tumors that are too large for endoscopic ablation,

- Grade 1-2 TaT1 disease.

The procedure can be performed using open, laparoscopic, or robotic techniques. It enables closure of defects approximately $4 \mathrm{~cm}$ in size. A $1 \mathrm{~cm}$ safety margin is resected on the proximal and distal aspects of the tumor. A double J stent is left in place for an average of $30-45$ days following anastomosis $(21,40)$. Distal segmental ureterectomy is recommended for patients with low stage/grade disease, those who are not eligible for endoscopic surgery, and when preserving renal function is necessary. To avoid tension on the anastomosis, the bladder can be suspended from the psoas muscle (psoas hitch) or a Boari flap can be made. In cases where anastomosis is not possible, the anastomosis can be achieved by forming a tube from a segment of ileum (Monti technique) (21).

Colin et al. (41) compared the 5-year outcomes of RNU (416 patients) and segmental ureterectomy (52 patients) in a multicenter, retrospective study. They reported that surgery type did not affect cancer-specific, recurrence-free, or metastasis-free survival. In selected patients, short-term oncologic outcomes indicate that segmental ureterectomy is also a valid method (41).

Retrograde ureteroscopic technique: First described by Goodman (42) in 1984. In terms of technique, using a rigid ureteroscope without a guide is recommended to avoid suspected tissue damage. Removing the tumor with forceps or basket and then applying laser [holmium:yttrium-aluminumgarnet (YAG)] or electrocautery to the base is recommended. Similarly, a flexible ureteroscope is used for upper ureteral and intrarenal evaluation and treatment (43), but anatomical obstructions (strictures and adhesions) may preclude performing the procedure exactly as described (44).

In their meta-analysis of 736 patients in 22 studies, Cutress et al. (44) reported 53\% recurrence, 15\% progression, 9\% diseasespecific mortality, and $19 \%$ subsequent NU with a follow-up period of 14-73 months. Complications were observed in 14\% of patients (78\% were strictures, others included perforation, sepsis, and hemorrhage) (44). Of the recent research examining the treatment efficacy of retrograde surgery, a study by Scotland et al. (45) is noteworthy. In their study, 80 patients with biopsy-proven low-grade UUTTs with a mean size of $3.04 \mathrm{~cm}$ were followed for a mean of $\mathbf{4 3 . 6}$ months after ureteroscopic laser ablation. All of the patients underwent cystoscopy and ureteroscopy every 3 months until tumor-free. Follow-up of tumor-free patients was done every 6 months for the first 5 years and yearly thereafter. During follow-up, ipsilateral recurrence was observed in $90.5 \%$ of the patients, with $31.7 \%$ progressing in grade at a mean of 26.3 months. Sixteen patients (20\%) underwent RNU after a mean of 23.2 months. Rates of overall and cancer-specific survival at 5 years were $75 \%$ and $84 \%$, respectively. It was concluded that ureteroscopic treatment of UUTTs $>2 \mathrm{~cm}$ in size is a treatment option that preserves organ 
function and offers an alternative to RNU, provided that the patient is closely monitored due to the risk of recurrence (45). Antegrade percutaneous technique: Technical advances have led to the abandonment of this method except for patients with diversion, solitary kidney, or anatomic obstacles (strictures, adhesions). Treatment using the antegrade approach is usually conducted in 3 sessions. Technically, renal access is achieved by the same steps used in percutaneous stone extraction surgery (ureteral catheter placement, prone position, access from the calyx at the angle most convenient for the procedure, and renax placement). The tumor is removed by excision or tearing, and the base is coagulated with YAG laser or electrocautery. Multiple access points may be required. NU is performed when peroperative frozen examination reveals invasive highgrade tumor (46). If the same pathology is encountered at any stage of conservative follow-up, the treatment is also NU (47). When peroperative pathology indicates a low-grade tumor, the procedure is concluded by placing a nephrostomy catheter. If there is suspicion of residual tumor, or a biopsy sample will be taken from the base, a second session is performed within the same week (46). If pathology is invasive at this stage, NU should be performed. If examination indicates tumor absence or lowgrade pathology and nephrostogram shows no extravasation/ obstruction, intrarenal mitomycin-C or BCG can be instilled (48). After 2-4 weeks of intrarenal chemo-immunotherapy, a third endoscopy is done and a biopsy sample is obtained. Follow-up begins after nephrostomy $(46,48)$.

Complications of percutaneous tumor extraction are similar to those of stone extraction surgery: fever, hemorrhage, and additional organ injuries. Furthermore, irrigation pressure must be kept below $40 \mathrm{~cm} \mathrm{H}_{2} \mathrm{O}$ to avoid tumor seeding due to backflow of the irrigation fluid. Using distilled water is recommended due to its cytolytic property (49). However, there remains a risk of tumor development along the access path and tumor extension due to urinary tract perforation despite all of these precautions (50).

Jarrett et al. (51) reported a recurrence rate of 33\% based on the 9 -year follow-up of 30 patients. Tumor grade has been reported to be a determinant of recurrence and survival (recurrence was $18 \%$ with $\mathrm{G} 1$ and $50 \%$ with G3). As a result, presence of a multifocal tumor $\geq 1.5 \mathrm{~cm}$ in size and the presence of dysplasia were defined as determinants of recurrence (52).

The recurrence rate in patients treated by percutaneous approach is $13-65 \%$. The rates of bladder recurrence, overall survival, and cancer-specific survival have been reported as $15-42 \%$, approximately $92 \%$, and $75-100 \%$, respectively (53). Previous studies retrospectively evaluating endoscopic and open surgical methods reported that $20 \%$ of patients treated endoscopically subsequently underwent RNU. It was also reported that in well-selected low-grade patients, cancerspecific survival was similar with endoscopic treatment and RNU, whereas RNU provided a survival advantage in patients with high-grade tumors (44). To determine correct tumor grade, the endoscopic biopsies and pathology specimens of patients who later underwent RNU were evaluated. There was $80 \%$ agreement between endoscopic and RNU pathology results. The study showed that with endoscopy, $25 \%$ of patients may have overlooked lesions, $50 \%$ of which contain undetectable CIS (54).
In another study, comparison of local UUTT patients who underwent endoscopic treatment and those who had RNU showed similar overall and cancer-specific survival rates. However, it is emphasized that further prospective, randomized, multicenter studies were needed to reliably compare oncologic outcomes (55).

Adjuvant therapies: $B C G$, mitomycin- $C$, epirubicin, thiotepa, and $\mathrm{BCG} /$ interferon have been used in the treatment of urothelial tumors of the UUT, both for primary CIS (56) and after resection and ablation $(57,58,59)$. However, due to insufficient patient numbers, it has not been possible to definitively demonstrate the efficacy of these methods (including mitomycin-C, BCG, and other agents) with scientific evidence $(60,61)$. Nevertheless, BCG is most commonly used after antegrade resection and mitomycin- $C$ is most commonly used after ureteroscopic resection (62). After ureteroscopic treatment, $60 \mathrm{mg}$ mitomycin-C is instilled for 1 hour once a week for 6 weeks using a ureteral catheter (pressure $\leq 20 \mathrm{~cm}$ $\mathrm{H}_{2} \mathrm{O}$ ) or directly into the bladder in cases with proven reflux $(63,56)$.

Following percutaneous resection, BCG therapy is usually recommended 1 week after the second follow-up nephroscopy (after ruling out extravasion and obstruction) and administered once a week for 6 weeks ( $360 \mathrm{mg} \mathrm{BCG/150} \mathrm{mL} \mathrm{saline,} \leq 20 \mathrm{~cm}$ $\mathrm{H}_{2} \mathrm{O}$ pressure and $1 \mathrm{~mL} / \mathrm{min}$ instillation rate, total of 2.5 hours per session) with intravenous antibiotic prophylaxis (64). In terms of the side effects of adjuvant therapy, the risk of systemic BCG sepsis is particularly noteworthy. Complete healing of the mucosa prior to administration (though not absolutely necessary for mitomycin-C) and low-pressure instillation are very important. Especially after percutaneous resection, routine use of isoniazid, close monitoring for sepsis, and waiting for 2 weeks have been suggested (62,65). A 2018 meta-analysis by Gregg et al. (66) including a total of 13 comparative studies is the most recent research on adjuvant therapies for UUTT. Ultimately, the authors emphasized the need for new therapeutic agents and more randomized clinical trials (66).

\section{Conclusion}

The gold standard in the treatment of UUTTs is RNU and cuff excision. Unlike the bladder, endoscopic interventions during follow-up require a higher level of technology and experience. Similar to bladder tumors, the local stage and histological grade of the tumor are the main prognostic factors $(67,68)$. For this reason, organ-sparing treatments are recommended for small, superficial, and low-grade tumors in both elective and nonelective procedures (69). The most common conservative technique is retrograde ureteroscopy. However, this procedure cannot be performed due to technical reasons in $25 \%$ of patients. An antegrade percutaneous route can be used in these cases and in patients with large intrarenal tumors.

Studies with larger patient numbers and longer follow-up periods are needed to better characterize the effects of adjuvant therapies on survival, recurrence, and prognosis. In the conservative approach, patient compliance with close monitoring is as important a factor as tumor pathology, size, and location. According to the schedule recommended in follow-up protocols, for the first 3 years patients should be 
evaluated with ureteroscopy every 6 months in addition to radiological and laboratory exams. If this is not considered feasible, NU and cuff excision should always be the first choice for treatment.

\section{Ethics}

Peer-review: Externally peer-reviewed.

Financial Disclosure: The authors declared that this study received no financial support.

\section{References}

1. Munoz JJ, Ellison LM. Upper tract urothelial neoplasms: incidence and survival during the last 2 decades. J Urol 2000;164:1523-1525.

2. Siegel R, Naishadham D, Jemal A. Cancer statistics, 2012. CA Cancer J Clin 2012;62:10-29.

3. Roupret $M$, Babjuk $M$, Böhle $A$, et al. Guidelines on Urothelial carcinomas of the upper urinary tract. In EAU Guidelines, edition presented at the EUA Annual Congress, Madrid 2016:1-28.

4. Raman SP, Fishman EK. Upper and Lower Tract Urothelial Imaging Using Computed Tomography Urography. Urol Clin North Am 2018;45:389-405

5. Bus MT, de Bruin DM, Faber D], et al. Optical diagnostics for upper urinary tract urothelial cancer: technology, thresholds, and clinical applications. J Endourol 2015;29:113-123.

6. Traxer O, Geavlete B, de Medina SG, et al. Narrow-band imaging digital flexible ureteroscopy in detection of upper urinary tract transitionalcell carcinoma: initial experience. J Endourol 2011;25:19-23.

7. Ahmad S, Aboumarzouk O, Somani B, et al. Oral 5-aminolevulinic acid in simultaneous photodynamic diagnosis of upper and lower urinary tract transitional cell carcinoma - a prospective audit. BJU Int 2012;110:596-600.

8. Aboumarzouk OM, Mains E, Moseley $H$, et al. Diagnosis of upper urinary tract tumours: is photodynamic diagnosis assisted ureterorenoscopy required as an addition to modern imaging and ureterorenoscopy? Photodiagnosis Photodyn Ther 2013;10:127-133.

9. Margulis V, Shariat SF, Matin SF, et al. Outcomes of radical nephroureterectomy: a series from the Upper Tract Urothelial Carcinoma Collaboration. Cancer 2009;115:1224-1233.

10. Novara G, De Marco V, Dalpiaz O, et al. Independent predictors of metachronous bladder transitional cell carcinoma (TCC) after nephroureterectomy for TCC of the upper urinary tract. BJU Int 2008;101:1368-1374.

11. Shariat SF, Favaretto RL, Gupta A, et al. Gender differences in radical nephroureterectomy for upper tract urothelial carcinoma. World J Urol 2011;29:481-486.

12. Rojas CP, Castle SM, Llanos CA, et al. Low biopsy volume in ureteroscopy does not affect tumor biopsy grading in upper tract urothelial carcinoma. Urol Oncol 2013;31:1696-1700.

13. Melamed MR, Reuter VE. Pathology and staging of urothelial tumors of the kidney and ureter. Urol Clin North Am 1993;20:333-347.

14. Jeldres $C$, Sun $M$, Isbarn $H$, et al. A population-based assessment of perioperative mortality after nephroureterectomy for upper-tract urothelial carcinoma. Urology 2010;75:315-320.

15. Rouprêt $M$, Colin $P$, Yates DR. A new proposal to risk stratify urothelial carcinomas of the upper urinary tract (UTUCs) in a predefinitive treatment setting: low-risk versus high-risk UTUCs. Eur Urol 2014;66:181-183.

16. Network N. NCCN Guidelines Version 4. 2018 Bladder Cancer. 2018; Available from: http//www.nccn.org/professionals/physician_gls/ bladder.pdf.

17. Rouprêt M, Babjuk M, Compérat $E$, et al. European guidelines on upper tract urothelial carcinomas: 2013 update. Eur Urol 2013;63:1059-1071.

18. Steinberg GD. Nephroureterectomy. In: Graham SD, Glenn JF, eds.
Glenn's urologic surgery, 5th ed. Philadelphia, New York LippincottRaven, 1998:147-155.

19. Clayman RV, Kavoussi LR, Figenshau RS, et al. Laparoscopic nephroureterectomy: initial clinical case report. J Laparoendosc Surg 1991;1:343-349.

20. Adibi M, Youssef R, Shariat SF, et al. Oncological outcomes after radical nephroureterectomy for upper tract urothelial carcinoma: comparison over the three decades. Int J Urol 2012;19:1060-1066.

21. Sagalowsky Al, Jarrett TW, Flanigan RC. Urothelial tumors of the upper urinary tract and ureter. In: Wein AJ, Kavoussi LR, Novick AC, Partin AW, Peters CA, eds. Campbell's Urology, 10th ed. New York, Sounders, 2012;1516-1553.

22. Sukumar S, Rogers CG. Robotic nephroureterectomy. In: John $\mathrm{H}$, Wiklund P, eds. Robotic urology, 2nd ed. New York, Springer, 2012;43-50.

23. Rassweiler JJ, Schulze M, Marrero R, et al. Laparoscopic nephroureterectomy for upper urinary tract transitional cell carcinoma: is it better than open surgery? Eur Urol 2004;46:690-697.

24. Zlotta AR. Should urologists always perform a bladder cuff resection during nephroureterectomy, and which method should they use? Eur Urol 2010;57:970-972.

25. Lughezzani G, Sun M, Perrotte P, et al. Should bladder cuff excision remain the standard of care at nephroureterectomy in patients with urothelial carcinoma of the renal pelvis? A population-based study. Eur Urol 2010;57:956-962.

26. Gill IS, Soble IJ, Miller SD, Sung GT. A novel technique for management of the en bloc bladder cuff and distal ureter during laparoscopic nephroureterectomy. J Urol 1999;161:430-434.

27. Zargar H, Krishnan J, Autorino R, et al. Robotic nephroureterectomy: a simplified approach requiring no patient repositioning or robot redocking. Eur Urol 2014;66:769-777.

28. Darwiche F, Swain S, Kallingal G, et al. Operative technique and early experience for robotic-assisted laparoscopic nephroureterectomy (RALNU) using da Vinci Xi. Springerplus 2015;4:298.

29. Tamhankar AS, Patil SR, Ahluwalia P, Gautam G. Robot-assisted radical nephroureterectomy with extended template lymphadenectomy for upper tract urothelial carcinoma: An outcome analysis. Indian J Urol 2018;34:212-218.

30. Stravodimos KG, Komninos C, Kural AR, Constantinides C. Distal ureterectomy techniques in laparoscopic and robot-assisted nephroureterectomy: Updated review. Urol Ann 2015;7:8-16.

31. Hanna N, Sun M, Trinh QD, et al. Propensity-score-matched comparison of perioperative outcomes between open and laparoscopic nephroureterectomy: a national series. Eur Urol 2012;61:715-721.

32. Li WM, Shen JT, Li CC, et al. Oncologic outcomes following three different approaches to the distal ureter and bladder cuff in nephroureterectomy for primary upper urinary tract urothelial carcinoma. Eur Urol 2010;57:963-969.

33. Cha EK, Shariat SF, Kormaksson M, et al. Predicting clinical outcomes after radical nephroureterectomy for upper tract urothelial carcinoma. Eur Urol 2012;61:818-825.

34. Raman JD, Ng CK, Scherr DS, et al. Impact of tumor location on prognosis for patients with upper tract urothelial carcinoma managed by radical nephroureterectomy. Eur Urol 2010;57:1072-1079.

35. Zou L, Zhang L, Zhang $\mathrm{H}$, et al. Comparison of post-operative intravesical recurrence and oncological outcomes after open versus laparoscopic nephroureterectomy for upper urinary tract urothelial carcinoma. World J Urol 2014;32:565-570.

36. Blackmur JP, Stewart GD, Egong EA, et al. Matched-pair analysis of open versus laparoscopic nephroureterectomy for upper urinary tract urothelial cell carcinoma. Urol Int 2015;94:156-162.

37. Stewart GD, Humphries KJ, Cutress ML, et al. Long-term comparative outcomes of open versus laparoscopic nephroureterectomy for 
upper urinary tract urothelial-cell carcinoma after a median followup of 13 years. J Endourol 2011;25:1329-1335.

38. Kondo T, Hashimoto $Y$, Kobayashi $H$, et al. Template-based lymphadenectomy in urothelial carcinoma of the upper urinary tract: impact on patient survival. Int J Urol 2010;17:848-854.

39. Lee HY, Yeh HC, Wu WJ, et al. The diagnostic ureteroscopy before radical nephroureterectomy in upper urinary tract urothelial carcinoma is not associated with higher intravesical recurrence. World J Surg Oncol 2018;16:135.

40. Simforoosh N, Mosapour E, Maghsudi R. Laparoscopic ureteral resection and anastomosis for management of low-grade transitionalcell carcinoma. J Endourol 2005; 19:287-289.

41. Colin P, OuzzaneA, Pignot G, etal. Comparison of oncological outcomes after segmental ureterectomy or radical nephroureterectomy in urothelial carcinomas of the upper urinary tract: results from a large French multicentre study. BJU Int 2012;110:1134-1141.

42. Goodman TM. Ureteroscopy with rigid instruments in the management of distal ureteral disease. J Urol 1984;132:250-253.

43. Grasso M, Fraiman M, Levine M. Ureteropyeloscopic diagnosis and treatment of upper urinary tract urothelial malignancies. Urology 1999;54:240-246.

44. Cutress ML, Stewart GD, Zakikhani P, et al. Ureteroscopic and percutaneous management of upper tract urothelial carcinoma (UTUC): systematic review. BJU Int 2012;110:614-628.

45. Scotland KB, Kleinmann N, Cason D, et al. Ureteroscopic Management of Large $\geq 2 \mathrm{~cm}$ Upper Tract Urothelial Carcinoma: A Comprehensive Twenty-three Year Experience. Urology 2018;18:30614-9.

46. Clark PE, Streem SB, Geisinger MA. 13-year experience with percutaneous management of upper tract transitional cell carcinoma. J Urol 1999;161:772-775.

47. Seisen T, Colin P, Rouprêt M. Risk-adapted strategy for the kidneysparing management of upper tract tumours. Nat Rev Urol 2015;12:155-166.

48. Liatsikos EN, Dinlenc CZ, Kapoor R, Smith AD. Transitional-cell carcinoma of the renal pelvis: ureteroscopic and percutaneous approach. J Endourol 2001;15:377-383.

49. Woodhouse CR, Kellett MJ, Bloom HJ. Percutaneous renal surgery and local radiotherapy in the management of renal pelvic transitional cell carcinoma. Br J Urol 1986;58:245-249.

50. Huang A, Low RK, deVere White R. Nephrostomy tract tumor seeding following percutaneous manipulation of a ureteral carcinoma. J Urol 1995; 153:1041-1042.

51. Jarrett TW, Sweetser PM, Weiss GH, Smith AD. Percutaneous management of transitional cell carcinoma of the renal collecting system: 9-year experience. J Urol 1995;154:1629-1635.

52. Patel A, Soonawalla P, Shepherd SF, et al. Long-term outcome after percutaneous treatment of transitional cell carcinoma of the renal pelvis. J Urol 1996;155:868-874.

53. Park BH, Jeon SS. Endoscopic management of upper urinary tract urothelial carcinoma. Korean J Urol 2013;54:426-432.
54. Yamany T, van Batavia J, Ahn J, et al. Ureterorenoscopy for upper tract urothelial carcinoma: how often are we missing lesions? Urology 2015;85:311-315.

55. Yakoubi R, Colin P, Seisen T, et al. Radical nephroureterectomy versus endoscopic procedures for the treatment of localised upper tract urothelial carcinoma: a meta-analysis and a systematic review of current evidence from comparative studies. Eur J Surg Oncol 2014;40:1629-1634.

56. Rastinehad AR, Smith AD. Bacillus Calmette-Guérin for upper tract urothelial cancer: is there a role? J Endourol 2009;23:563-568.

57. Goel MC, Mahendra V, Roberts JG. Percutaneous management of renal pelvic urothelial tumors: long-term followup. J Urol 2003; 169:925-929.

58. Van Helsdingen PJ, Rikken CH. Treatment of urothelial carcinoma of the upper urinary tract following prostatocystectomy with mitomycin C instillation in the ileal loop. J Urol 1986;136:461-463.

59. Katz MH, Lee MW, Gupta M. Setting a new standard for topical therapy of upper-tract transitional-cell carcinoma: BCG and interferon-alpha2B. J Endourol 2007;21:374-377.

60. Rastinehad AR, Ost MC, Vanderbrink BA, et al. A 20-year experience with percutaneous resection of upper tract transitional carcinoma: is there an oncologic benefit with adjuvant bacillus Calmette Guérin therapy? Urology 2009;73:27-31.

61. Cutress ML, Stewart GD, Wells-Cole S, et al. Long-term endoscopic management of upper tract urothelial carcinoma: 20-year singlecentre experience. BJU Int 2012;110:1608-1617.

62. Tawfiek ER, Bagley DH. Upper-tract transitional cell carcinoma. Urology 1997;50:321-329.

63. Ho KL, Chow GK. Ureteroscopic resection of upper-tract transitionalcell carcinoma. J Endourol 2005;19:841-848.

64. Thalmann GN, Markwalder R, Walter B, Studer UE. Long-term experience with bacillus Calmette-Guerin therapy of upper urinary tract transitional cell carcinoma in patients not eligible for surgery. J Urol 2002;168:1381-1385.

65. Hayashida Y, Nomata K, Noguchi M, et al. Long-term effects of bacille Calmette-Guérin perfusion therapy for treatment of transitional cell carcinoma in situ of upper urinary tract. Urology 2004;63:1084-1088.

66. Gregg RW, Vera-Badillo FE, Booth CM, et al. Perioperative chemotherapy for urothelial carcinoma of the upper urinary tract: A systematic review and meta-analysis. Crit Rev Oncol Hematol 2018; 128:58-64.

67. Charbit L, Gendreau MC, Mee S, Cukier J. Tumors of the upper urinary tract: 10 years of experience. J Urol 1991;146:1243-1246.

68. Ozsahin M, Zouhair A, Villà S, et al. Prognostic factors in urothelial renal pelvis and ureter tumours: a multicentre Rare Cancer Network study. Eur J Cancer 1999;35:738-743.

69. McCarron JP Jr, Chasko SB, Gray GF Jr. Systematic mapping of nephroureterectomy specimens removed for urothelial cancer: pathological findings and clinical correlations. J Urol $1982 ; 128: 243-246$. 